Journal of Applied AnALysis

Vol. 11, No. 1 (2005), pp. 63-79

\title{
THE NOTION OF $\boldsymbol{V}$ - $r$-INVEXITY IN DIFFERENTIABLE MULTIOBJECTIVE PROGRAMMING
}

\author{
T. ANTCZAK \\ Received October 1, 2002 and, in revised form, March 31, 2003
}

\begin{abstract}
In this paper, a generalization of convexity, namely $V-r$ invexity, is considered in the case of nonlinear multiobjective programming problems where the functions involved are differentiable. The assumptions on Pareto solutions are relaxed by means of $V$ - $r$-invex functions. Also some duality results are obtained for such optimization problems.
\end{abstract}

\section{Introduction}

An important concept in mathematical models in economics, game theory, optimal control, and decision theory is that of a vector minimum or Pareto optimum.

In the recent years, optimization problems with vector-valued cost criteria or programming problems with several objectives conflicting with one another have been of considerable interest, particularly in economics.

2000 Mathematics Subject Classification. 90C29, 26B25, 90C26.

Key words and phrases. Multiobjective programming, (weak) Pareto optimal solution, (strictly) $V$-r-invex function with respect to $\eta$, optimality conditions, duality.

ISSN 1425-6908（C) Heldermann Verlag. 
The convexity of functions occurring in multiobjective programming problems is usually assumed to be sufficient condition in seeking Pareto optimal solutions (see, for example, [14], [16], [19], [25], [27]). To relax convexity assumptions imposed in theorems on sufficient optimality conditions and duality, various generalized convexity notions have been proposed (see, for example, [2], [3], [4], [12], [15], [17], [21], [23]).

An invex function is one of the generalized convex functions and this was introduced by Hanson [13]. He considered a differentiable function $f: X \rightarrow \mathbb{R}, X \subset \mathbb{R}^{n}$, for which there exists an $n$-dimensional vector function $\eta: X \times X \rightarrow \mathbb{R}^{n}$ such that, for all $x, u \in X$, the inequality

$$
f(x)-f(u) \geq \nabla f(u) \eta(x, u)
$$

holds. In [9], Craven called functions satisfying (1) invex. Of course, differentiable convex functions are invex with respect to the function $\eta(x, u)=$ $x-u$. A simple characterization of invexity was given for both constrained and unconstrained optimization problems by Ben-Israel and Mond [7]. After the works of Hanson and Craven, other types of differentiable functions have appeared with the intent of generalizing invex functions from different points of view. One of such generalizations is $r$-invexity which was introduced by Antczak [4]. Many authors have studied some properties and further generalizations of scalar Hanson's functions to vector-valued functions, in view of applications to multiobjective optimization problems.

Egudo and Hanson [12] have studied a multiobjective problem with Wolfetype and Mond-Weir-type duals for invex objective and quasi-invex constraint functions. Weir [27] considered a multi-objective programming problem involving invex functions and obtained Kuhn-Tucker necessary and sufficient conditions for a feasible point to be a Pareto optimal solution. Optimization and duality results for a vector optimization problem with invex set-valued data are also given, for example, in [18], [26]. In [1], Antczak introduced a new class of differentiable nonconvex vector-valued functions, namely $(p, r)$-invex vector-valued functions with respect to $\eta$. This class of functions is a generalization of scalar $(p, r)$-invex functions [2] to the vectorial case. This fact leads to consider more general definition than Hanson's definition (1).

Recently, Jeyakumar and Mond [15] have observed that one major difficulty in all of these extensions of convexity is that invex problems require the same functions $\eta$ for the objective and constraint functions. This requirement turns out to be a major restriction in applications. To improve upon this situation they have defined $V$-invex functions and its various generalizations. They have obtained optimality conditions for differentiable multiobjective programming problems and established some duality results. 
Basing on the definition of $V$-invex vector-valued functions and the definition of scalar $r$-invex functions [4], we introduce a new class of differentiable nonconvex vector valued functions, namely $V$ - $r$-invex functions with respect to $\eta$.

However, the main purpose of this article is to apply $V$-r-invexity to develop optimality conditions and duality theory for the differentiable multiobjective programming problems. Considering the concept of a (weak) Pareto solution in multiobjective programming problems in which the functions occurring belong to the class of $V$ - $r$-invex functions, we give some sufficient conditions for optimality. As in the scalar case (see [2]), the concept of $V$-r-invexity function plays an important role. A dual of the Mond-Weir type, the Wolfe type and the mixed type are also considered and a number of duality results are established. Duality results are established by using a Pareto type relation between the primal and dual objective functions and the concept of $V$-r-invexity.

\section{New classes of nonconvex vector-valued functions}

Let $\mathbb{R}^{n}$ denote the $n$-dimensional Euclidean space and $\mathbb{R}_{+}^{n}$ its non-negative orthant.

The following convention for equalities and inequalities will be used throughout the paper.

For any $x=\left(x_{1}, x_{2}, \ldots, x_{n}\right)^{T}, y=\left(y_{1}, y_{2}, \ldots, y_{n}\right)^{T}$, we define:

(i) $x=y$ if and only if $x_{i}=y_{i}$ for all $i=1,2, \ldots, n$;

(ii) $x>y$ if and only if $x_{i}>y_{i}$ for all $i=1,2, \ldots, n$;

(iii) $x \geqq y$ if and only if $x_{i} \geq y_{i}$ for all $i=1,2, \ldots, n$;

(iv) $x \geq y$ if and only if $x \geqq y$ and $x \neq y$.

Throughout the paper, we will use the same notation for row and column vectors when the interpretation is obvious.

Hanson defined invex functions which allow the use of the Kuhn-Tucker conditions as sufficient conditions for optimality in constrained optimization problems. Moreover, Wolfe weak duality holds between primal problem and its associated Wolfe dual problem under invexity assumption.

However, the major difficulty is that the invex problems require the same function $\eta$ restriction in applications for the objective function and the constraints. This requirement turns out to be severe restriction in applications.

Now, we show how this situation can be improved and how can be extended the class of invex functions to posses the two well-known properties for constrained optimization problems (that is, the sufficiency of KuhnTucker conditions and Wolfe weak duality). The following definition generalizes the definition of a class of $r$-invex functions [2] to the case of a class of $V$-r-invex functions. 
Definition 1. Let $S$ be a nonempty open subset of $\mathbb{R}^{n}$ and let $r$ be an arbitrary real number. A differentiable function $f: S \rightarrow \mathbb{R}^{k}$ is called a $V$ $r$-invex function (a strictly $V$-r-invex function) with respect to $\eta$ at $u \in S$ on $S$ if, there exist functions $\eta: S \times S \rightarrow \mathbb{R}^{n}$ and $\alpha: S \times S \rightarrow \mathbb{R}_{+}^{k} \backslash\{0\}$ such that for each $x \in S$, the relation

$$
\begin{array}{ll}
\frac{1}{r} e^{r f(x)} \geqq \frac{1}{r} e^{r f(u)}[1+r \alpha(x, u) \nabla f(u) \eta(x, u)] & (>\text { if } x \neq u) \text { for } r \neq 0, \\
f(x)-f(u) \geqq \alpha(x, u) \nabla f(u) \eta(x, u) & (>\text { if } x \neq u) \text { for } r=0
\end{array}
$$

holds.

If inequalities (2) are satisfied at any point $u \in S$, then $f$ is said to be $V$-r-invex (strictly $V$-r-invex) with respect to $\eta$ on $S$.

It should be pointed out that the exponentials appearing on the left-hand sides of inequalities (2) are understood to be taken componentwise.

Equivalently, the definition of $V$-r-invexity of function $f$ can be written in the following form:

Definition 2. A differentiable function $f: S \rightarrow \mathbb{R}^{k}$ is called an $\alpha_{i}$-r-invex function (a strictly $\alpha_{i}$-r-invex function) with respect to $\eta$ at $u \in S$ on $S$ if, there exist functions $\eta: S \times S \rightarrow \mathbb{R}^{n}$ and $\alpha_{i}: S \times S \rightarrow \mathbb{R}_{+} \backslash\{0\}, i=1, \ldots, k$, such that for each $x \in S$, the relation

$$
\begin{array}{ll}
\frac{1}{r} e^{r f_{i}(x)} \geqq \frac{1}{r} e^{r f_{i}(u)}\left[1+r \alpha_{i}(x, u) \nabla f_{i}(u) \eta(x, u)\right] & (>\text { if } x \neq u) \text { for } r \neq 0, \\
f_{i}(x)-f_{i}(u) \geqq \alpha_{i}(x, u) \nabla f_{i}(u) \eta(x, u) & (>\text { if } x \neq u) \text { for } r=0
\end{array}
$$

holds.

If inequalities (3) are satisfied at any point $u \in S$, then $f$ is said to be $\alpha_{i}$-r-invex (strictly $\alpha_{i}-r$-invex) with respect to $\eta$ on $S$.

Note that asymmetry between Definition 1 and Definition 2. The first one exhibits only one positive factor $\alpha$ which however (in a vector optimization problem) may be different with respect to the objective and the constraint functions.

Remark 3. In order to define an analogous class of (strictly) $V$-r-incave functions with respect to $\eta$, the direction of the inequalities in (2) should be changed to the opposite one.

Remark 4. If, for all $x, u \in S, \alpha_{i}(x, u) \equiv 1, i=1, \ldots, k$, then the above definition reduces to the usual definition of $r$-invexity (see [1]). 
Remark 5. In the case when $\eta(x, u)=x-u$ we obtain a definition of $V$-r-convexity (for $k=1$ Definition 1 reduces to the definition of a scalar $r$-convex function, see [5]).

Now, we consider an example of a $V$-r-invex function.

Example 6. Let $f: \mathbb{R} \rightarrow \mathbb{R}^{2}$ be a differentiable vector-valued function defined by

$$
f(x)=\left(\log \left(x^{2}+1\right), \log \left(x^{2}-2 x+5\right)\right) .
$$

It is not difficult to prove, by Definition 1 or Definition 2 , that $f$ is $V$-1-invex on $\mathbb{R}$ with respect to $\eta$ defined by

$$
\eta(x, u)= \begin{cases}-\frac{1}{u-1} & \text { if } u<0 \\ -1 & \text { if } u \geq 0\end{cases}
$$

where $\alpha=\left(\alpha_{1}, \alpha_{2}\right): \mathbb{R} \times \mathbb{R} \rightarrow \mathbb{R}^{2}$ is defined by

$$
\begin{aligned}
& \alpha_{1}(x, u)= \begin{cases}\frac{1}{2} u(u-1) & \text { if } u<0, \\
\frac{1}{2} u & \text { if } u \geq 0,\end{cases} \\
& \alpha_{2}(x, u)= \begin{cases}\frac{1}{2}\left(u^{2}-2 u+5\right) & \text { if } u<0, \\
\frac{1}{2} & \text { if } 0 \leq u<1, \\
2 k-1 & \text { if } 2 k-1 \leq u<2 k+1, \quad k=1,2, \ldots\end{cases}
\end{aligned}
$$

But $f$ is neither a $V$-invex function on $\mathbb{R}$ with respect to $\eta$ and with $\alpha$ defined above (see [15]) nor an $r$-invex vector-valued function on $\mathbb{R}$ with respect to the function $\eta$ (see Remark 4).

Proposition 7. Let $f: \mathbb{R}^{n} \rightarrow \mathbb{R}^{k}$ be a $V$-r-invex ( $V$-r-incave) vector function with respect to $\eta$ on $\mathbb{R}^{n}$. The following propositions are true:

a) If a be any real number, then the function $f+a$ is $V-r$-invex $(V-r$ incave) with respect to $\eta$ on $\mathbb{R}^{n}$.

b) If $\lambda$ is any positive real number, then the function $\lambda f$ is $V$-r $/ \lambda$-invex ( $V$-r $/ \lambda$-incave) with respect to $\eta$ on $\mathbb{R}^{n}$.

c) $f$ is $V$-r-invex ( $V$-r-incave) with respect to $\eta$ on $\mathbb{R}^{n}$ if and only if $-f$ is $V$-(-r)-incave $\left(V-(-r)\right.$-invex) with respect to $\eta$ on $\mathbb{R}^{n}$. 


\section{Optimality conditions in multiobjective programming}

In general, a multiobjective programming problem is formulated as the following vector minimization problem:

$$
\begin{gathered}
f(x)=\left(f_{1}(x), f_{2}(x), \ldots, f_{k}(x)\right) \rightarrow \min \\
g(x) \leqq 0, \\
h(x)=0, \\
x \in X,
\end{gathered}
$$

where $f_{i}: X \rightarrow \mathbb{R}, i \in K=\{1, \ldots, k\}, g_{j}: X \rightarrow \mathbb{R}, j \in J=\{1, \ldots, m\}$, $h_{s}: X \rightarrow \mathbb{R}, s \in Q=\{1, \ldots, q\}$ are differentiable functions on a nonempty open set $X \subset \mathbb{R}^{n}$.

Let $D=\{x \in X: g(x) \leqq 0, h(x)=0\}$ be the set of feasible solutions for problem (CVP).

Definition 8. A vector optimization problem (CVP) is said to be a $V$ $r$-invex multiobjective programming problem if each of the functions $f_{i}$, $i \in K, g_{j}, j \in J, h_{s}, s \in Q$ are $V$-r-invex functions with respect to the same function $\eta$ on $D$, but the positive factor which however may be different with respect to the objective and the constraint functions.

Unlike problems with a unique objective, in which there may exist an optimal solution to the effect that it minimizes the objective function, in vector optimization problem there does not necessarily exist a point which may be optimal for all objectives. It is known that for a problem of this type, the concept of optimal solution found in single-objective optimization problem (so-called scalar optimization problem) is not valid. The concept of an ideal point - one that minimizes each objective - is in general not feasible. The solution concept of a multiobjective programming problem (CVP) are referred to in the literature as efficient solutions, or Paretooptimal. Other variants include weakly efficient solutions, local efficient solutions, etc.

For such optimization problems minimization means in general obtaining (weak) Pareto optimal solutions in the following sense [24] (also see [17]):

Definition 9. A feasible point $\bar{x}$ is said to be a Pareto solution (efficient solution) for (CVP) if and only if there exists no $x \in D$ such that

$$
f(x) \leq f(\bar{x}) .
$$


Definition 10. A feasible point $\bar{x}$ is said to be a weak Pareto solution (weakly efficient solution, weak minimum) for (CVP) if and only if there exists no $x \in D$ such that

$$
f(x)<f(\bar{x})
$$

Speaking roughly, a point $\bar{x} \in D$ is Pareto optimal in (CVP) if and only if we can improve (in the sense of minimization) the value of one of the objective functions only at the cost of making at least one of the remaining objective functions worse; it is weak Pareto optimal if and only if we cannot improve further all of the objective functions simultaneously.

The following result gives the relationship between the vector minimization problem (CVP) and the corresponding scalar minimization problem.

Lemma 11 ([8]). A point $\bar{x} \in D$ is a Pareto optimal solution for (CVP) if and only if $\bar{x}$ is a solution of the following problems for each $i \in K$ :

$$
\begin{gathered}
f_{i}(x) \rightarrow \min \\
\text { s.t. } x \in D, \quad f_{s}(x) \leq f_{s}(\bar{x}), \quad \text { for all } s \in K \backslash\{i\} .
\end{gathered}
$$

In [10], Craven obtained the necessary optimality conditions for a multiobjective programming problem (CVP). Thus, under a suitable constraint qualification [6], [20], the following generalized Kuhn-Tucker conditions are true:

Theorem 12. Let $\bar{x}$ be a Pareto minimal point (a weak Pareto minimal point) in problem (CVP). Moreover, we assume that $g$ and $h$ satisfy a suitable constraint qualification at $\bar{x}[6],[20]$. Then there exist $\bar{\lambda} \in \mathbb{R}^{k}, \bar{\xi} \in \mathbb{R}^{m}$, $\bar{\mu} \in \mathbb{R}^{q}$ such that

$$
\begin{gathered}
\bar{\lambda} \nabla f(\bar{x})+\bar{\xi} \nabla g(\bar{x})+\bar{\mu} \nabla h(\bar{x})=0, \\
\bar{\xi} g(\bar{x})=0, \\
\bar{\lambda} \in \mathbb{R}_{+}^{k}, \bar{\lambda} \neq 0, \bar{\xi} \in \mathbb{R}_{+}^{m}, \quad \bar{\mu} \in \mathbb{R}_{+}^{q} .
\end{gathered}
$$

Let $J(z):=\left\{j \in J: g_{j}(z)=0\right\}$ denote the active constraint index set at some fixed point $z \in D$.

Next, we point out role played by $V$-r-invexity to state the sufficiency of the above Kuhn-Tucker conditions. Thus, we obtained results which extended Jeyakumar's results [15].

It is well known [25] that for such multiobjective optimization problems, under convexity assumptions, the Kuhn-Tucker conditions (5)-(7) are also sufficient for (weak) Pareto optimality. Now, we show that assumptions of convexity (or invexity) may be replaced by $V$-r-invexity. 
Theorem 13. Let $\bar{x}$ be a feasible point for (CVP) and let $f_{i}, i \in K$, be $\alpha_{i}-r$-invex with respect to $\eta$ at $\bar{x}$ on $D, g_{j}, j \in J(\bar{x})$, be $\beta_{j}-r$-invex with respect to $\eta$ at $\bar{x}$ on $D$ and $h_{s}, s \in Q$, be $\gamma_{s}-r$-invex with respect to $\eta$ at $\bar{x}$ on D. Further, we assume that there exist $\bar{\lambda} \in \mathbb{R}_{+}^{k}, \bar{\xi}_{J(\bar{x})} \in \mathbb{R}_{+}^{J(\bar{x})}, \bar{\mu} \in \mathbb{R}_{+}^{q}$, $\left(\bar{\lambda} \geq 0, \bar{\xi}_{J(\bar{x})} \geqq 0, \bar{\mu} \geqq 0\right), \bar{\lambda}>0, \bar{\xi}_{J(\bar{x})} \geqq 0, \bar{\mu} \geqq 0$, such that:

$$
\begin{gathered}
\left(\bar{\lambda} \nabla f(\bar{x})+\bar{\xi}_{J(\bar{x})} \nabla g_{J(\bar{x})}(\bar{x})+\bar{\mu} \nabla h(\bar{x})\right) \eta(x, \bar{x}) \geqq 0, \\
\bar{\xi} g(\bar{x})=0 .
\end{gathered}
$$

Then $\bar{x}$ is a (weak) Pareto optimal point for (CVP).

Proof. We proceed by contradiction. Suppose that $\bar{x}$ is not a Pareto optimal point for $(\mathrm{CVP})$, that is, there exists $\widetilde{x} \in D$ such that

$$
f(\widetilde{x})-f(\bar{x}) \leq 0 .
$$

By assumption, $f_{i}, i \in K$, are $\alpha_{i}$-r-invex with respect to the same function $\eta$ at $\bar{x}$ on $D$. By Definition 2, we have

$$
\frac{1}{r} e^{r f_{i}(\widetilde{x})} \geqq \frac{1}{r} e^{r f_{i}(\bar{x})}\left[1+r \alpha_{i}(\widetilde{x}, \bar{x}) \nabla f_{i}(\bar{x}) \eta(\widetilde{x}, \bar{x})\right] .
$$

From definition $\alpha_{i}(\widetilde{x}, \bar{x})>0, i \in K$, and since $\bar{\lambda}>0$, we obtain

$$
\frac{1}{r} \frac{\bar{\lambda}_{i}}{\alpha_{i}(\widetilde{x}, \bar{x})}\left(e^{r\left(f_{i}(\widetilde{x})-f_{i}(\bar{x})\right)}-1\right) \geqq \bar{\lambda}_{i} \nabla f_{i}(\bar{x}) \eta(\widetilde{x}, \bar{x}),
$$

and so

$$
\frac{1}{r} \sum_{i=1}^{k} \frac{\bar{\lambda}_{i}}{\alpha_{i}(\widetilde{x}, \bar{x})}\left(e^{r\left(f_{i}(\widetilde{x})-f_{i}(\bar{x})\right)}-1\right) \geqq \sum_{i=1}^{k} \bar{\lambda}_{i} \nabla f_{i}(\bar{x}) \eta(\widetilde{x}, \bar{x}) .
$$

Hence, by (10),

$$
0 \geq \bar{\lambda} \nabla f(\bar{x}) \eta(\widetilde{x}, \bar{x})
$$

By assumption, $g_{j}, j \in J(\bar{x})$, are $\beta_{j}$-r-invex with respect to the same function $\eta$ at $\bar{x}$ on $D$. By Definition 2, we have

$$
\frac{1}{r} e^{r g_{j}(\widetilde{x})} \geqq \frac{1}{r} e^{r g_{j}(\bar{x})}\left[1+r \beta_{i}(\widetilde{x}, \bar{x}) \nabla g_{J(\bar{x})}(\bar{x}) \eta(\widetilde{x}, \bar{x})\right], \quad j \in J(\bar{x}) .
$$

From definition $\beta_{j}(\widetilde{x}, \bar{x})>0$, and since $\bar{\xi}_{j}>0, j \in J(\bar{x})$, we obtain

$$
\begin{aligned}
& \frac{1}{r} \sum_{j \in J(\bar{x})} \frac{\bar{\xi}_{j}}{\beta_{j}(\widetilde{x}, \bar{x})}\left(e^{(r / \bar{\xi})_{j}\left(\bar{\xi}_{j} g_{j}(\widetilde{x})-\bar{\xi}_{j} g_{j}(\bar{x})\right)}-1\right) \\
& \geqq \sum_{j \in J(\bar{x})} \bar{\xi}_{j} \nabla g_{j}(\bar{x}) \eta(\widetilde{x}, \bar{x}) .
\end{aligned}
$$


Hence, using (9) together with a feasibility of $\widetilde{x}$, we get

$$
\frac{1}{r} \sum_{j \in J(\bar{x})} \frac{\bar{\xi}_{j}}{\beta_{j}(\widetilde{x}, \bar{x})}\left(e^{(r / \bar{\xi})_{j}\left(\bar{\xi}_{j} g_{j}(\widetilde{x})-\bar{\xi}_{j} g_{j}(\bar{x})\right)}-1\right) \leqq 0 .
$$

Thus, by (12)

$$
0 \geqq \bar{\xi}_{J(\bar{x})} \nabla g_{J(\bar{x})}(\bar{x}) \eta(\widetilde{x}, \bar{x}) .
$$

Analogously, by assumption, $h_{s}, s \in Q$, be $\gamma_{s}-r$-invex with respect to $\eta$ at $\bar{x}$ on $D$. By Definition 2, we have

$$
\frac{1}{r} e^{r h_{s}(\widetilde{x})} \geqq \frac{1}{r} e^{r h_{s}(\bar{x})}\left[1+r \gamma_{i}(\widetilde{x}, \bar{x}) \nabla h_{s}(\bar{x}) \eta(\widetilde{x}, \bar{x})\right], \quad s \in Q .
$$

Since $\gamma_{s}(\widetilde{x}, \bar{x})>0, s \in Q$, and $\bar{\mu}_{s} \geqq 0$, then

$$
\frac{1}{r} \sum_{s \in Q} \frac{\bar{\mu}_{s}}{\gamma_{s}(\widetilde{x}, \bar{x})}\left(e^{r\left(h_{s}(\widetilde{x})-h_{s}(\bar{x})\right)}-1\right) \geqq \sum_{s \in Q} \bar{\mu}_{s} \nabla h_{s}(\bar{x}) \eta(\widetilde{x}, \bar{x}) .
$$

and by $\widetilde{x}, \bar{x} \in D$

$$
0 \geqq \sum_{s \in Q} \bar{\mu}_{s} \nabla h_{s}(\bar{x}) \eta(\widetilde{x}, \bar{x}) .
$$

Adding (11), (13), (14) we obtain the inequality

$$
0 \geq\left(\bar{\lambda} \nabla f(\bar{x})+\bar{\xi}_{J(\bar{x})} \nabla g_{J(\bar{x})}(\bar{x})+\bar{\mu} \nabla h(\bar{x})\right) \eta(\widetilde{x}, \bar{x}) .
$$

which contradicts the Kuhn-Tucker condition (5).

Proof for a weak Pareto optimality is analogous.

Now, we present an example of a $V$-r-invex multiobjective programming problem. Moreover, we show that the assumption of the positivity of the Lagrange multiplier $\bar{\lambda}$ is required to prove the efficiency of $\bar{x}$; to prove the weak efficiency of $\bar{x}$ it is sufficient to assume that the Lagrange multiplier $\bar{\lambda}$ is only nonnegative.

Example 14. We consider the following multiobjective programming problem

$$
\begin{aligned}
& f(x)=\left(\log \left(x_{1}^{2}+1\right), \log \left(x_{2}^{2}+1\right)\right) \\
& g(x)=\left(\log \left(\frac{1}{2} x_{1}^{2}+\frac{1}{2}\right), \log \left(\frac{1}{2} x_{2}^{2}+\frac{1}{2}\right)\right) \leqq 0 .
\end{aligned}
$$

For the above problem,

$$
\begin{aligned}
D & =\left\{\left(x_{1}, x_{2}\right) \in \mathbb{R}^{2}: g(x) \leqq 0\right\} \\
& =\left\{\left(x_{1}, x_{2}\right) \in \mathbb{R}^{2}:-1 \leqq x_{1} \leqq 1 \wedge-1 \leqq x_{2} \leqq 1\right\}
\end{aligned}
$$


It is not difficult to prove, by Definition 1 or Definition 2, that the considered multiobjective programming problem is $V$-1-invex on $D$ with respect to $\eta=\left(\eta_{1}, \eta_{2}\right): D \times D \rightarrow \mathbb{R}^{2}$ defined by

$$
\eta_{i}(x, u)=\left\{\begin{array}{ll}
\frac{1}{2} & \text { if } u_{i}<0, \\
-1 & \text { if } u_{i} \geq 0
\end{array} \quad i=1,2\right.
$$

where $\alpha=\left(\alpha_{1}, \alpha_{2}\right): D \times D \rightarrow \mathbb{R}_{+}^{2}$ is defined by

$$
\alpha_{i}(x, u)= \begin{cases}-u_{i} & \text { if } u_{i}<0 \\ 1 & \text { if } u_{i}=0, \quad i=1,2 \\ \frac{1}{2} u_{i} & \text { if } u_{i}>0\end{cases}
$$

and $\beta=\left(\beta_{1}, \beta_{2}\right): D \times D \rightarrow \mathbb{R}_{+}^{2}$ is defined by

$$
\beta_{i}(x, u)=\left\{\begin{array}{ll}
-\frac{1}{u_{i}} & \text { if } u_{i}<0, \\
1 & \text { if } u_{i} \geqq 0,
\end{array} \quad i=1,2 .\right.
$$

Now, at the point $\widetilde{x}=(0,1)$, condition (8) of Theorem 13 takes the following form

$$
\left(\bar{\lambda}_{2}+2 \bar{\xi}_{2}\right)(-1) \geqq 0,
$$

and we have to take

$$
\bar{\lambda}_{2}=0, \quad \bar{\xi}_{2}=0 .
$$

But $\widetilde{x}=(0,1)$ is not the Pareto optimal solution in the considered problem (it is only the weak Pareto solution) since $f((0,1))=(0, \log 2) \geq$ $(0,0)=f((0,0))$. It is not difficult to see that there no $x \in D$ such that $f(x) \leq f((0,0))$. Moreover, at the point $\bar{x}=(0,0)$ from the condition (8) of Theorem 13 follows that we can take $\bar{\lambda}_{i}>0, i=1,2$. This means that $\bar{x}=(0,0)$ is the Pareto optimal solution in the considered multiobjective optimization problem.

Now, we establish the sufficient condition for optimality in (CVP) under $V$-r-invexity of the Lagrangian.

Theorem 15. Let $\bar{x}$ be a feasible point for (CVP) and we assume the following:

i) $f, g, h$ are differentiable at $\bar{x}$, 
ii) there exist $\bar{\lambda} \in \mathbb{R}_{+}^{k}, \bar{\xi}_{J(\bar{x})} \in \mathbb{R}_{+}^{J(\bar{x})}, \bar{\mu} \in \mathbb{R}_{+}^{q},\left(\bar{\lambda} \geq 0, \bar{\xi}_{J(\bar{x})} \geqq 0, \bar{\mu} \geqq 0\right)$ $\bar{\lambda}>0, \bar{\xi}_{J(\bar{x})} \geqq 0, \bar{\mu} \geqq 0$, such that:

$$
\left(\bar{\lambda} \nabla f(\bar{x})+\bar{\xi}_{J(\bar{x})} \nabla g_{J(\bar{x})}(\bar{x})+\bar{\mu} \nabla h(\bar{x})\right) \eta(x, \bar{x}) \geqq 0, \quad \forall x \in D .
$$

iii) the Lagrangian $\bar{\lambda} f+\bar{\xi}_{J(\bar{x})} g_{J(\bar{x})}+\bar{\mu} h$ is $V$-r-invex with respect to $\eta$ at $\bar{x}$ on $D$.

Then $\bar{x}$ is a (weak) Pareto optimal point for (CVP).

Proof. We proceed by contradiction. Suppose that $\bar{x}$ is not a Pareto optimal point for (CVP), that is, there exists $\widetilde{x} \in D$ such that

$$
f(\widetilde{x})-f(\bar{x}) \leq 0 .
$$

Since $\bar{x} \in D$ and $\widetilde{x} \in D$, by hypothesis (ii) there exist $\bar{\lambda}>0, \bar{\xi}_{J(\bar{x})} \geqq 0$, $\bar{\mu} \geqq 0$, such that (15) is fulfilled. Moreover, from the feasibility of $\bar{x}$ and $\widetilde{x}$ in problem (CVP) together with $\bar{\lambda}>0, \bar{\xi} \geq 0, \bar{\mu} \geq 0$ follows

$0 \geq \bar{\lambda}(f(\widetilde{x})-f(\bar{x})), 0 \geqq \bar{\xi}_{J(\bar{x})}\left(g_{J(\bar{x})}(\widetilde{x})-g_{J(\bar{x})}(\bar{x})\right), \quad 0=\bar{\mu}(h(\widetilde{x})-h(\bar{x}))$.

Hence,

$$
\bar{\lambda} f(\bar{x})+\bar{\xi}_{J(\bar{x})} g_{J(\bar{x})}(\bar{x})+\bar{\mu} h(\bar{x}) \geq \bar{\lambda} f(\widetilde{x})+\bar{\xi}_{J(\bar{x})} g_{J(\bar{x})}(\widetilde{x})+\bar{\mu} h(\widetilde{x}) .
$$

Since $\bar{\lambda} f+\bar{\xi}_{J(\bar{x})} g_{J(\bar{x})}+\bar{\mu} h$ is $V$-r-invex with respect to $\eta$ at $\bar{x}$, by (17), we get the inequality

$$
0 \geq\left(\bar{\lambda} \nabla f(\bar{x})+\bar{\xi} \nabla g_{J(\bar{x})}(\bar{x})+\bar{\mu} \nabla h(\bar{x})\right) \eta(\widetilde{x}, \bar{x}),
$$

which is a contradiction to the assumption (ii) of the theorem.

Proof for weak Pareto optimality is similar.

\section{Mond-Weir duality}

Now, in relation to (CVP) we consider the following multiobjective dual problem, which is in the format of Mond-Weir [22] duality:

$$
\begin{gathered}
f(y)=\left(f_{1}(y), f_{2}(y), \ldots, f_{k}(y)\right) \rightarrow \max \\
(\lambda \nabla f(y)+\xi \nabla g(y)+\mu \nabla h(y)) \eta(x, y) \geqq 0, \forall x \in D, \\
\xi g(y)+\mu h(y) \geqq 0, \\
y \in X, \\
\lambda \in \mathbb{R}_{+}^{k}, \lambda \geq 0, \lambda \mathbf{e}=\mathbf{1}, \\
\xi \in \mathbb{R}_{+}^{m}, \xi \geqq 0, \\
\mu \in \mathbb{R}_{+}^{q}, \mu \geqq 0,
\end{gathered}
$$


where $\mathbf{e}=(\mathbf{1}, \ldots, \mathbf{1}) \in \mathbb{R}^{\mathbf{k}}$.

Let $W$ denote the set of all feasible points of (MWVD) and $I(z)$ the set of objective functions indices for which a corresponding multipler is positive at the point $z$, that is, $I(z):=\left\{i \in K: \lambda_{i}>0\right\}$. Moreover, we denote by $\operatorname{pr}_{X} W$ the projection of the set $W$ on $X$.

Now, we give some useful lemma which a simple proof will be omitted.

Lemma 16. Let $(y, \lambda, \xi, \mu)$ be a feasible point for (MWVD). Moreover, we assume one of the following conditions:

a) $\xi g+\mu h$ is a $V$-r-invex function with respect to $\eta$ at $y$ on $D \cup \operatorname{pr}_{X} W$,

b) $g_{j}, j \in J(y)$, are $\beta_{j}-r$-invex with respect to $\eta$ at $y$ on $D \cup \operatorname{pr}_{X} W$ and $h_{s}, s \in Q$, are $\gamma_{s}$-r-invex with respect to $\eta$ at $y$ on $D \cup \operatorname{pr}_{X} W$.

Then

$$
(\xi \nabla g(y)+\mu \nabla h(y)) \eta(x, y) \leqq 0, \quad \forall x \in D
$$

Theorem 17 (Weak duality). Let $x$ and $(y, \lambda, \xi, \mu)$ be feasible points for $(C V P)$ and $(M W V D)$, respectively. Moreover, we assume that $f_{i}, i \in I(y)$, are $\alpha_{i}$-r-invex with respect to the same function $\eta$ at $y$ on $D \cup \operatorname{pr}_{X} W, g_{j}$, $j \in J(y)$, are $\beta_{j}$-r-invex with respect to $\eta$ at $y$ on $D \cup \operatorname{pr}_{X} W, h_{s}, s \in Q$, are $\gamma_{s}$-r-invex with respect to $\eta$ at $y$ on $D \cup \operatorname{pr}_{X} W$. Then $f(x) \nless f(y)$.

Proof. We consider only the case $r \neq 0$. We proceed by contradiction. Suppose that $f(x)<f(y)$. Since $f_{i}, i \in I(y)$, are $\alpha_{i}$-r-invex with respect to the same function $\eta$ at $y$ on $D \cup \operatorname{pr}_{X} W$, then by Definition 2,

$$
\frac{\lambda_{i}}{r}\left(e^{r\left(f_{i}(x)-f_{i}(y)\right)}-1\right) \geqq \lambda_{i} \alpha_{i}(x, y) \nabla f_{i}(y) \eta(x, y), \quad i \in I(y) .
$$

Using $f(x)<f(y)$, we get

$$
0>\lambda_{i} \alpha_{i}(x, y) \nabla f_{i}(y) \eta(x, y), \quad i \in I(y) .
$$

Since by definition $\alpha_{i}(x, y)>0, i=1, \ldots, k$, then we obtain

$$
0>\lambda \nabla f(y) \eta(x, y) .
$$

By assumption, $g_{j}, j \in J(y)$, are $\beta_{j}$-r-invex with respect to $\eta$ at $y$ on $D \cup \operatorname{pr}_{X} W$ and $h_{s}, s \in Q$, are $\gamma_{s}-r$-invex with respect to $\eta$ at $y$ on $D \cup \operatorname{pr}_{X} W$. Then, by Corollary 16, the inequality (18) holds. After adding both sides of (19) and (18), we obtain, for all $x \in D$, the inequality

$$
(\lambda \nabla f(y)+\xi \nabla g(y)+\mu \nabla h(y)) \eta(x, y)<0,
$$

which contradicts the constraint of (MWVD).

Now, we prove weak duality in the sense of Mond-Weir under $V$-r-invexity assumption imposed on the Lagrange function. 
Theorem 18 (Weak duality). Let $x$ and $(y, \lambda, \xi, \mu)$ be feasible points for (CVP) and (MWVD), respectively. Moreover, we assume that $\lambda f+\xi g+\mu h$ is $V$-r-invex with respect to $\eta$ at $y$ on $D \cup \operatorname{pr}_{X} W$. Then $f(x) \nless f(y)$.

Proof. We proceed by contradiction. Suppose that

$$
f(x)<f(y) .
$$

Since $\lambda f+\xi g+\mu h$ is $V$-r-invex with respect to $\eta$ at $y$ on $D \cup \operatorname{pr}_{X} W$, it follows that the following inequality

$$
\begin{aligned}
& \frac{1}{r}\left(e^{r(\lambda f(x)+\xi g(x)+\mu h(x)-(\lambda f(y)+\xi g(y)+\mu h(y)))}-1\right) \\
& \geqq \alpha(x, y)(\lambda \nabla f(y)+\xi \nabla g(y)+\mu \nabla h(y)) \eta(x, y)
\end{aligned}
$$

holds for all $x \in D$. Using (20) together with the feasibility of $x$ and $(y, \lambda, \xi, \mu)$ for $(\mathrm{CVP})$ and (MWVD), respectively, we obtain

$$
0>\frac{1}{r}\left(e^{r(\lambda f(x)+\xi g(x)+\mu h(x)-(\lambda f(y)+\xi g(y)+\mu h(y)))}-1\right),
$$

and by (21)

$$
0>\alpha(x, y)(\lambda \nabla f(y)+\xi \nabla g(y)+\mu \nabla h(y)) \eta(x, y), \quad \forall x \in D
$$

Since by definition $\alpha(x, y)>0$, it follows a contradiction to the dual constraint

$$
(\lambda \nabla f(y)+\xi \nabla g(y)+\mu \nabla h(y)) \eta(x, y) \geqq 0, \text { for all } x \in D \text {. }
$$

Theorem 19 (Strong duality). Let $\bar{x}$ be a (weak) Pareto solution in (CVP) at which a suitable constraint qualification is satisfied. Then there exist $\bar{\lambda} \in \mathbb{R}_{+}^{k}, \bar{\lambda} \neq 0, \bar{\xi} \in \mathbb{R}_{+}^{m}, \bar{\mu} \in \mathbb{R}_{+}^{q}$, such that $(\bar{x}, \bar{\lambda}, \bar{\xi}, \bar{\mu})$ is feasible for (MWVD) with $\bar{\xi} g(\bar{x})=0$. If also weak duality Theorem 17 (or Theorem 18) holds then $(\bar{x}, \bar{\lambda}, \bar{\xi}, \bar{\mu})$ is a (weak) maximum for (MWVD), and the objective function values are equal in problems (CVP) and (MWVD).

Proof. From assumption, $\bar{x}$ is Pareto optimal solution in (CVP). Hence, by Theorem 12 , there exist $\bar{\lambda} \in \mathbb{R}_{+}^{k}, \bar{\lambda} \neq 0, \bar{\xi} \in \mathbb{R}_{+}^{m}, \bar{\xi} \geqq 0, \bar{\mu} \in \mathbb{R}_{+}^{q}, \bar{\mu} \geqq 0$, such that the Kuhn-Tucker conditions (5)-(7) hold. It follows from (5)-(7) that $(\bar{x}, \bar{\lambda}, \bar{\xi}, \bar{\mu})$ is feasible in (MWVD). Also, by weak duality (Theorem 17 or Theorem 18), it follows that $(\bar{x}, \bar{\lambda}, \bar{\xi}, \bar{\mu})$ is optimal for (MWVD).

Theorem 20 (Converse duality). Let $(\bar{y}, \bar{\lambda}, \bar{\xi}, \bar{\mu})$ be a weak maximum for $(M W V D)$ and $\bar{\lambda} f+\bar{\xi} g+\bar{\mu} h$ be $V$-r-invex with respect $\eta$ at $\bar{y}$ on $D \cup \operatorname{pr}_{X} W$. Then $\bar{y}$ is weak Pareto optimal in (CVP). 
Proof. This follows on the line of the proof of Theorem 18.

\section{Wolfe duality}

Relative to problem (CVP) we consider the following Wolfe dual:

$$
\begin{gathered}
\varphi(y, \lambda, \xi, \mu)=f(y)+\xi g(y)+\mu h(y) \rightarrow \max \\
(\lambda \nabla f(y)+\xi \nabla g(y)+\mu \nabla h(y)) \eta(x, y) \geqq 0, \forall x \in D, \\
y \in X, \\
\lambda \in \mathbb{R}_{+}^{k}, \lambda \geq 0, \lambda \mathbf{e}=\mathbf{1}, \\
\xi \in \mathbb{R}_{+}^{m}, \xi \geqq 0, \\
\mu \in \mathbb{R}_{+}^{q}, \bar{\mu} \geqq 0 .
\end{gathered}
$$

Let $\widetilde{W}$ denote the set of all feasible points of (WVD).

Theorem 21 (Weak duality). Let $x$ and $(y, \lambda, \xi, \mu)$ be feasible points for $(C V P)$ and $(W V D)$, respectively. If $\lambda f+\xi g+\mu h$ is $V$-r-invex with respect to $\eta$ at $y$ on $D \cup \operatorname{pr}_{X} \widetilde{W}$. Then $f(x) \nless \varphi(y, \lambda, \xi, \mu)$.

Proof. We assume that $f(x)<\varphi(y, \lambda, \xi, \mu)$ and exhibit a contradiction. Because $\xi \in \mathbb{R}_{+}^{m}, \mu \in \mathbb{R}_{+}^{q}$, and $x \in D$, we get from the inequality above that

$$
f(x)+\xi g(x)+\mu h(x)<f(y)+\xi g(y)+\mu h(y) .
$$

Since $\lambda \geq 0$ and $\lambda \mathbf{e}=\mathbf{1}$ we obtain

$$
\lambda f(x)+\xi g(x)+\mu h(x)<\lambda f(y)+\xi g(y)+\mu h(y) .
$$

Using $V$-r-invexity of $\lambda f+\xi g+\mu h$ with respect to $\eta$ on $D \cup \operatorname{pr}_{X} \widetilde{W}$ together with (23), we get the inequality

$$
(\lambda \nabla f(y)+\xi \nabla g(y)+\mu \nabla h(y)) \eta(x, y)<0,
$$

contradicting the dual constraint of (WVD).

Theorem 22 (Strong duality). Let $\bar{x}$ be a (weak) Pareto optimal in (CVP) at which a suitable constraint qualification is satisfied. Then there exist $\bar{\lambda} \in \mathbb{R}_{+}^{k}, \bar{\lambda} \neq 0, \bar{\xi} \in \mathbb{R}_{+}^{m}, \bar{\mu} \in \mathbb{R}_{+}^{q}$, such that $(\bar{x}, \bar{\lambda}, \bar{\xi}, \bar{\mu})$ is feasible for (WVD) and the objective functions of $(C V P)$ and (WVD) are equal at these points. If also, weak duality (Theorem 21) between (CVP) and (WVD) holds then $(\bar{x}, \bar{\lambda}, \bar{\xi}, \bar{\mu})$ is a (weak) maximum in (WVD). 
Proof. Since $\bar{x}$ satisfies all conditions of the Theorem 12, there exist $\bar{\lambda} \in$ $\mathbb{R}_{+}^{k}, \bar{\lambda} \neq 0, \bar{\xi} \in \mathbb{R}_{+}^{m}, \bar{\xi} \geqq 0, \bar{\mu} \in \mathbb{R}_{+}^{q}, \bar{\mu} \geqq 0$, such that the Kuhn-Tucker conditions (5)-(7) hold. By (5)-(7), we have that $(\bar{x}, \bar{\lambda}, \bar{\xi}, \bar{\mu})$ is feasible for (WVD). Also, by Theorem 21 , it follows that $(\bar{x}, \bar{\lambda}, \bar{\xi}, \bar{\mu})$ is optimal for (WVD).

Theorem 23 (Converse duality). Let $(\bar{y}, \bar{\lambda}, \bar{\xi}, \bar{\mu})$ be a weak maximum for (WVD) such that $\bar{y} \in D$. Moreover, we assume that $\varphi(y, \bar{\lambda}, \bar{\xi}, \bar{\mu})$ is $V-r$ invex with respect $\eta$ at $\bar{y}$ on $D \cup \operatorname{pr}_{X} \widetilde{W}$. Then $\bar{y}$ is weak Pareto optimal in (CVP).

Proof. We suppose that $\bar{y}$ is not a weak minimum for (CVP). Then, there exists $\widetilde{x} \in D$ such that $f(\widetilde{x})<f(\bar{y})$. The feasibility of $(\bar{y}, \bar{\lambda}, \bar{\xi}, \bar{\mu})$ in (WVD) implies that $\bar{\xi} g(\bar{y})=0, \bar{\mu} h(\bar{y})=0$, and also the feasibility of $\widetilde{x}$ in (CVP) implies that $\bar{\xi} g(\widetilde{x}) \leqq 0, \bar{\mu} h(\widetilde{x})=0$. Since $\bar{\lambda} \in \mathbb{R}_{+}^{k}$ and $\bar{\lambda} \mathbf{e}=\mathbf{1}$, and by $f(\widetilde{x})<f(\bar{y})$ we get

$$
\bar{\lambda} f(\widetilde{x})+\bar{\xi} g(\widetilde{x})+\bar{\mu} h(\widetilde{x})<\bar{\lambda} f(\bar{y})+\bar{\xi} g(\bar{y})+\bar{\mu} h(\bar{y}) .
$$

Since $\bar{\lambda} f+\bar{\xi} g+\bar{\mu} h$ is $V$-r-invex with respect $\eta$ at $\bar{y}$ for all $x \in D \cup \operatorname{pr}_{X} \widetilde{W}$, by (24), we get

$$
(\bar{\lambda} \nabla f(\bar{y})+\bar{\xi} \nabla g(\bar{y})+\bar{\mu} \nabla h(\bar{y})) \eta(\widetilde{x}, \bar{y})<0 .
$$

This contradicts the dual constraints of problem (WVD), which holds at $\bar{y}$ for all $x \in D$, and also for $\widetilde{x}$.

\section{Mixed duality}

In a similar manner to that given in [22], relative to problem (CVP), we consider the following multiobjective dual problem:

$$
\begin{gathered}
\varphi(y, \lambda, \xi, \mu)=f(y)+\xi g(y)+\mu h(y) \rightarrow \max \\
(\lambda \nabla f(y)+\xi \nabla g(y)+\mu \nabla h(y)) \eta(x, y) \geqq 0, \forall x \in D, \\
\xi g(y)+\mu h(y) \geqq 0, \\
y \in X, \\
\lambda \in \mathbb{R}_{+}^{k}, \lambda \geq 0, \lambda \mathbf{e}=\mathbf{1}, \\
\xi \in \mathbb{R}_{+}^{m}, \xi \geqq 0, \\
\mu \in \mathbb{R}_{+}^{q}, \mu \geqq 0 .
\end{gathered}
$$

Since the set of all feasible solutions for problem (MVD) is the same as the set of feasible solutions for problem (MWVD) we denote it by $W$. 
Proofs for corresponding duality results for the above dual run on the same lines as the proofs of the Theorems 18-20 and they were be omitted in this work.

Theorem 24 (Weak duality). Let $x$ and $(y, \lambda, \xi, \mu)$ be feasible points for $(C V P)$ and (MVD), respectively. If the Lagrangian $\varphi(y, \lambda, \xi, \mu)$ is $V-r$ invex with respect to $\eta$ at $y$ on $D \cup \operatorname{pr}_{X} W$. Then $f(x) \nless \varphi(y, \lambda, \xi, \mu)$.

Theorem 25 (Strong duality). Let $\bar{x}$ be a Pareto optimal or a weak Pareto optimal for (CVP) at which a suitable constraint qualification [6] is satisfied. Then there exist $\bar{\lambda} \in \mathbb{R}_{+}^{k}, \bar{\lambda} \neq 0, \bar{\xi} \in \mathbb{R}_{+}^{m}, \bar{\xi} \geqq 0, \bar{\mu} \in \mathbb{R}_{+}^{q}, \bar{\mu} \geqq 0$, such that $(\bar{x}, \bar{\lambda}, \bar{\xi}, \bar{\mu})$ is feasible for (MVD) and the objective functions of (CVP) and (MVD) are equal at these points. If, also, weak duality (Theorem 24) between (CVP) and (MVD) holds then $(\bar{x}, \bar{\lambda}, \bar{\xi}, \bar{\mu})$ is a (weak) maximum for (MVD).

Theorem 26 (Converse duality). Let $(\bar{y}, \bar{\lambda}, \bar{\xi}, \bar{\mu})$ be a weak maximum for (MVD) such that $\bar{y} \in D$. Moreover, we assume that the Lagrangian $\varphi(y, \bar{\lambda}, \bar{\xi}, \bar{\mu})$ is $V$-r-invex with respect $\eta$ at $\bar{y}$ on $D \cup \operatorname{pr}_{X} W$. Then $\bar{y}$ is weak Pareto optimal in (CVP).

\section{References}

[1] Antczak, T., Multiobjective programming with $(p, r)$-invexity, Zeszyty Nauk. Politech. Rzeszowskiej Mat. 25 (2001), 5-30.

[2] Antczak, T., (p,r)-invex sets and functions, J. Math. Anal. Appl. 263 (2001), 355379 .

[3] Antczak, T., On (p,r)-invexity-type nonlinear programming problems, J. Math. Anal. Appl. 264 (2001), 382-397.

[4] Antczak, T., r-pre-invexity and r-invexity in mathematical programming, Comput. Math. Appl., (to be published).

[5] Avriel, M., r-convex functions, Math. Program. 2 (1972), 309-323.

[6] Bazaraa, M. S., Sherali, H. D., Shetty, C. M., Nonlinear Programming: Theory and Algorithms, John Wiley and Sons, New York, 1991.

[7] Ben-Israel, A., Mond, B., What is invexity?, J. Austral. Math. Soc. Ser. B 28 (1986), $1-9$.

[8] Chankong, V., Haimes, Y. Y., Multiobjective Decision Making: Theory and Methodology, North-Holland, New York, 1983.

[9] Craven, B. D., Invex functions and constrained local minima, Bull. Austral. Math. Soc. 24 (1981), 357-366.

[10] Craven, B. D., Vector-valued optimization, in "Generalized Concavity in Optimization and Economics", S. Schaible and W. Ziemba (eds.), Academic Press, New York, 1981, 661-687. 
[11] Craven, B. D., Glover, B. M., Invex functions and duality, J. Austral. Math. Soc. Ser. A 39 (1985), 1-20.

[12] Egudo, R. R., Hanson, M. A., Multi-objective duality with invexity, J. Math. Anal. Appl. 126 (1987), 469-477.

[13] Hanson, M. A., On sufficiency of the Kuhn-Tucker conditions, J. Math. Anal. Appl. 80 (1981), 545-550.

[14] Jahn, J., Scalarization in multiobjective optimization, in "Mathematics of Multiobjective Optimization", CISM Courses and Lectures 289, Springer, Vienna, 1985, $45-88$.

[15] Jeyakumar, V., Mond, B., On generalized convex mathematical programming, J. Austral. Math. Soc. Ser. B 34 (1992), 43-53.

[16] Kanniappan, P., Necessary conditions for optimality of nondifferentiable convex multiobjective programming, J. Optim. Theory Appl. 40 (1983), 167-174.

[17] Luc, D. T., Theory of Vector Optimization, Lecture Notes in Econom. and Math. Systems 319, Springer-Verlag, Berlin, 1988.

[18] Luc, D. T., Malivert, C., Invex optimization problems, Bull. Austral. Math. Soc. 46 (1992), 47-66.

[19] Luc, D. T., Vector optimization, Lecture delivered at the summer school "Generalized Convexity and Monotonicity", August 1999, Samos, Greece.

[20] Mangasarian, O. L., Nonlinear Programming, McGraw-Hill, New York-LondonSydney, 1969.

[21] Martin, D. H., The essence of invexity, J. Optim. Theory Appl. 47 (1985), 65-76.

[22] Mond, B., Weir, T., Generalized concavity and duality, in "Generalized Concavity in Optimization and Economics", S. Schaible and W. T. Ziemba (eds.), Academic Press, New York, 1981, 263-279.

[23] Osuna-Gomez, R., Beato-Moreno, A., Rufian-Lizana, A., Generalized convexity in multiobjective programming, J. Math. Anal. Appl. 233 (1999), 205-220.

[24] Pareto, V., Cours de Économie Politique, Rouge, Lausanne, Switzerland, 1896, 1897.

[25] Singh, C., Optimality conditions in multiobjective differentiable programming, J. Optim. Theory Appl. 53 (1987), 115-123.

[26] Srivastava, M. K., Vector valued lagrangian and invexity, Opsearch 31 (1995), 266286.

[27] Weir, T., A note on invex functions and duality in multiple objective optimization, Opsearch 25 (1988), 98-104.

[28] Wolfe, P., A duality theorem for nonlinear programming, Quart. Appl. Math. 19 (1961), 239-244.

TADEUSZ ANTCZAK

FaCUlty of Mathematics

UNIVERSITY OF ŁÓDŹ

BANACHA 22

90-238 ŁÓDŹ, POLAND

E-MAIL: ANTCZAK@MATH.UNI.LODZ.PL 